\title{
Multilinguales
}

11 | 2019

Varia

\section{Textes littéraires et interculturalité en classe de FLE : enjeux et approches didactiques}

Literary texts and interculturality : issues and didactic approaches

Fatah Abdelouhab

\section{(2) OpenEdition}

Journals

Édition électronique

URL : http://journals.openedition.org/multilinguales/3860

DOI : $10.4000 /$ multilinguales.3860

ISSN : 2335-1853

Éditeur

Université Abderrahmane Mira - Bejaia

Référence électronique

Fatah Abdelouhab, «Textes littéraires et interculturalité en classe de FLE : enjeux et approches didactiques », Multilinguales [En ligne], 11 | 2019, mis en ligne le 17 juillet 2019, consulté le 02 juin 2020. URL : http://journals.openedition.org/multilinguales/3860 ; DOI : https://doi.org/10.4000/ multilinguales.3860

Ce document a été généré automatiquement le 2 juin 2020.

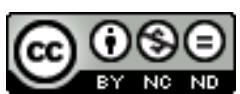

Multilinguales est mise à disposition selon les termes de la Licence Creative Commons Attribution Pas d'Utilisation Commerciale - Pas de Modification 4.0 International 


\title{
Textes littéraires et interculturalité en classe de FLE : enjeux et approches didactiques
}

\author{
Literary texts and interculturality: issues and didactic approaches
}

\author{
Fatah Abdelouhab
}

1 Le texte littéraire a connu divers traitements au fil des évolutions des méthodologies d'enseignement du FLE. Ayant connu la gloire et la déchéance, le texte littéraire a fini par devenir l'allié incontournable des nouvelles méthodes d'enseignement du FLE, quel que soit son degré de sophistication. En effet, les vertus du support littéraire le rehaussent et font de lui un outil de premier rang lors de l'acquisition d'une langue étrangère ou bien l'enseignement de la littérature.

2 Sur le plan didactique, le texte littéraire est le plus souvent appréhendé par les enseignants dans sa seule dimension instrumentale. Alors que la démarche didactique qui s'inscrit dans une perspective interculturelle procède de manière à situer, chaque texte littéraire, dans son contexte et à signaler l'enjeu interculturel de la lecture.

Considérant l'œuvre littéraire comme une production culturelle et tirant les conséquences de cette nature dans l'acte même de la lecture, le texte littéraire se métamorphose en un lieu où se côtoient langue(s) et culture(s). Ce support littéraire, baptisé « document authentique » depuis les apports de l'approche communicative, de par sa nature de produit anthropologique, constitue une source intarissable pour la rencontre de l'Autre. Ainsi, la littérature accorde la possibilité d'étudier l'être humain dans sa complexité, sa profondeur et surtout sa vulnérabilité.

4 Nous posons les questions suivantes: l'enseignant du moyen adhère-t-il à une didactique interculturelle qui initie à l'universalité et au vivre ensemble? Quels enjeux didactico-pédagogiques sont-ils impliqués dans la didactisation de l'interculturalité par le biais du support littéraire ? C'est à ces questions que nous tentons d'apporter des éléments de réponse en prenant appui sur les résultats d'une analyse des extraits littéraires insérés dans les quatre manuels du moyen. Nous présentons dans cet article 
un descriptif de l'analyse menée suivi de l'exposition des résultats et de leur interprétation.

Il est à souligner que le texte littéraire constitue le médium où se déploie l'interculturalité par excellence. L'œuvre littéraire peut donc constituer une voie d'accès aux différents codes sociaux et à des visions du monde dans la mesure où elle représente une mosaïque assez expressive du désir de soi et de l'autre. À ce propos, M. Abdallah-Pretceille affirme que

le texte littéraire, production de l'imaginaire, représente un genre inépuisable pour

l'exercice artificiel de la rencontre avec l'Autre ; rencontre par procuration certes,

mais rencontre tout de même » $(1999,2)$.

6 Le texte littéraire renferme souvent une représentation du monde, des valeurs partagées par deux cultures, encore faut-il savoir comment mettre en exergue cet héritage commun par une pratique interculturelle du texte littéraire.

7 Dans ces conditions, nous avons adopté l'approche anthropologique comme démarche car elle permet à l'apprenant-lecteur de se découvrir comme être de culture, identique ou différent de l'être de culture qu'est l'auteur. Par ailleurs, l'auteur porte un regard anthropologique particulier sur des représentations culturelles fondamentales, le végétal, l'animal, l'espace et le temps, telles qu'elles s'expriment dans les textes littéraires français ou francophones.

8 Ainsi, en nous inspirant de l'approche anthropologique proposée par L. Collès (1994) et celle des universels-singuliers, phénomènes culturels présents dans toute société mais que chacun traite à sa manière spécifique, proposée par L. Porcher (1987), notre objectif est de rechercher le général à travers le singulier. Il s'agit, d'une méthode d'analyse fondée sur l'universel-singulier. L'auteur, comme moi-social particulier, intègre en luimême l'ensemble du monde de son époque, il se constitue en emblème du monde, exprime l'universalité au sein de sa seule singularité.

9 Les analyses effectuées nous ont permis d'admettre que l'application de l'approche interculturelle aux faits littéraires, accroît, chez l'apprenant, une certaine conscience anthropologique par rapport aux faits humains auxquels il se trouve confronté, le rend aussi capable d'interpréter et de rassembler des signes délimités et de leur prêter des significations pertinentes.

\section{Le potentiel des textes littéraires}

10 L'enseignement du FLE par le texte littéraire vise des objectifs spécifiques qui ne sont pas toujours ceux de la formation littéraire en langue maternelle. Un texte littéraire en tant que texte authentique à part entière influence la personnalité de l'apprenant au niveau cognitif, affectif et esthétique. Par ailleurs, les professeurs peuvent se plaindre des contraintes induites par l'usage des textes littéraires en classe. En effet, la lecture littéraire en classe est caractérisée par un certain nombre de contraintes : le temps, le lieu, les objectifs définis par l'institution et le programme... etc. Cependant, la plupart des experts à l'instar de L. Porcher, M. Abdallah-Prétceille, A. Séoud, H. Besse, I. Gruca sont de l'avis selon lequel la littérature jouit d'une place indéniable dans l'enseignement des langues étrangères. En effet, les innombrables possibilités d'exploitation des textes littéraires viennent surtout de leur dimension socio- culturelle et esthétique qui les distingue d'autres textes sans valeur esthétique ce qui nous permet 
de développer et de cultiver la sensibilité esthétique et langagière de l'apprenantlecteur.

11 Eu égard au rôle primordial que joue la littérature dans la construction intellectuelle de l'apprenant-lecteur, du citoyen, de l'être humain, elle retrouve ses lettres de noblesse comme support didactique incontournable dans l'enseignement/apprentissage du FLE. D'après A. Dakhia :

Il ressort ainsi que le texte littéraire est un univers interculturel par excellence. La littérature y joue le rôle de passeur d'une culture à une autre à la condition expresse qu'on maintienne une distance respectueuse à la fois vis-à-vis de la culture de l'émetteur (celle qui l'a vue naître) et de la culture du récepteur (celle qu'assure la survie de l'œuvre) (Dakhia Abdelouahab, 2005 : 197).

12 En effet, chaque lecteur trouvera son compte dans l'œuvre littéraire indépendamment de son origine géographique, linguistique et culturelle. En d'autres termes, chaque lecteur empruntera la voix/voie qui lui permettra la découverte de la forêt vierge du sens absolu dans la forêt-texte que représente l'univers romanesque, en reprenant l'analogie introduite par A. Dakhia (2005 : 199). À propos du caractère polysémique et atemporel propres au texte littéraire, A. Séoud (1997 souligne :

13 Le texte littéraire, par définition, dépasse toute contingence, celle de l'ici et maintenant. L'écrivain écrit pour l'éternité, et Henri Besse fait remarquer pertinemment qu'on ne lit pas un journal vieux d'un an mais on prend plaisir à lire tel écrivain de l'Antiquité. Cette qualité, le texte littéraire la doit à sa polysémie, à sa richesse inépuisable de sens, qui fait que, par-delà l'espace et le temps, par-delà même parfois les frontières de la langue, il peut parler à tout le monde. Et à ce titre bien sûr, tout le monde, chaque lecteur peut légitimement, à la lecture, l'investir de ses propres sentiments, le voir, le vivre, le comprendre à sa façon (A. Séoud 1997 : 15).

De ce qui précède, A. Séoud aboutit à la conclusion selon laquelle « la littérature, le contact avec les textes, grâce à leur polysémie, est le terrain le plus favorable, le plus propice à l'expression interculturelle ». (A. Séoud, 1997 : 15)

15 Le contenu d'un texte littéraire nous permet de traiter des questions de société et d'enter en contact avec l'Autre. Le texte littéraire est le médiateur dans la rencontre et la (re) découverte de l'Autre. Il permet donc de saisir l'homme dans sa complexité, sa diversité et sa variabilité. La littérature " empêche les hommes d'être indifférents aux hommes » (Eugène Ionesco, Cité par A. Dakhia, 2005).

16 Ainsi, le support littéraire se révèle être une des plus sûres voies du dialogue entre les cultures puisque les textes, conçus comme point de rencontre d'univers différents, constituent des révélateurs privilégiés des visions plurielles du monde. À la fois, le texte littéraire trouve sa place dans l'enseignement de la langue comme dans celui de la culture " parce qu'il est l'un des lieux où s'élaborent et se transmettent les mythes et les rites dans lesquels une société se reconnaît et se distingue des autres " (H. Besse, $1989:$ 7). En outre, grâce à sa polysémie et à sa dimension universelle, le texte littéraire devient capable de " parler à tout le monde, par delà le temps et l'espace ».

La littérature est donc, avant tout, de la langue, destinée non seulement à des usages spécifiques mais également à des visées symboliques. Dans ce sens, M. AbdallahPretceille et L. Porcher insistent également sur la langue qui est la source et la " matière première » de la littérature qui serait, selon eux » [...] un tissu de phrases et de mots, une chair linguistique vivante et qui fonde l'humanité de l'homme." 
L'originalité de cette définition se traduit par la nature » vivante » et » humaniste » assignée à la littérature.

La littérature nait donc des mots et de l'usage diversifié (subjectif, objectif, etc.) que l'on en fait. C'est un lieu spécifique où l'homme, par le biais de la langue, exprime sa vision du monde. C'est également un espace où se rencontrent l'imaginaire et la réalité, le rêve et le vécu, l'identité et l'altérité, le Moi et l'Autre, en bref l'« humanité de l'homme ». La littérature permet donc, selon M. Abdallah- Pretceille et L. Porcher $(1996: 138)$.

[...] d'étudier l'homme dans sa complexité et sa variabilité. C'est cette dimension humaniste qui curieusement ressurgit et est directement interpellée en didactique des cultures ainsi qu'en formation générale.

De ce point de vue, le texte littéraire se présente comme la voie royale qui mène à la culture de soi et la culture d'autrui. Elle traduit l'universel, en offrant l'expérience du divers.

De ce qui précède, on affiche une volonté de mettre l'accent sur le rôle éminent du texte littéraire dans la motivation à la lecture, mais aussi à une langue et à une culture, car on a bien tort de passer sous silence cet immense réservoir que constitue le texte littéraire.

\section{Statut des textes littéraires dans les manuels du cycle moyen}

\subsection{Modalités de travail}

S'interroger sur le statut attribué aux documents littéraires dans les manuels du cycle moyen nous a amené à opter, dans un premier temps, pour une analyse des contenus. C'est pourquoi nous avons élaboré une grille de « balayage » des manuels.

Dans notre analyse, nous procédons comme suit :

1. Inventorier le nombre de textes littéraires insérés dans les manuels.

2. répartir l'ensemble des textes selon des critères significatifs (auteur, récurrence (nombre d'extraits)...).

3. Répartition des auteurs en fonction de leur origine (français ou francophones).

4. Classement des textes littéraires en fonction de leur genre.

\subsection{Corpus étudié}

Notre étude porte sur quatre manuels en usage dans le cycle moyen répartis comme suit :

Tableau 1 : Nombre des manuels dans le cycle moyen

\begin{tabular}{|l|l|l|l|l|l|}
\hline Cycle moyen & $1^{\text {ère }}$ & $2^{\text {ème }}$ & $3^{\text {ème }}$ & $4^{\text {ème }}$ & Total \\
\hline Nombre de Manuels & 1 & 1 & 1 & 1 & 4 \\
\hline
\end{tabular}

Notons que les manuels en usage au niveau du cycle moyen sont issus de la réforme éducative des programmes et des méthodes en 2003. Le français est érigé en première 
langue étrangère et son enseignement se fait dès la $3^{\text {ème }}$ année primaire. À cela s'ajoute la réhabilitation de la littérature dans les manuels de FLE notamment au moyen.

\subsection{Dimension didactique du texte littéraire} l'avènement de l'approche par compétences. Désormais, le texte littéraire est appréhendé comme réservoir d'informations socioculturelles en plus de sa qualité de laboratoire du langage. En effet, le texte littéraire constitue un espace de contact de langues et de cultures. R. Barthes, 1978, affirme à ce propos :

$\mathrm{Si}$, je ne sais par quel excès de socialisme ou de barbarie, toutes nos disciplines devraient être expulsées de l'enseignement sauf une, c'est la discipline littéraire qui devrait être sauvée, car toutes les sciences sont présentes dans le monument littéraire. (R. Barthes cité par J. L. Dufays- L. Gemenne et D. Ledure, 2005 : 129)

M. Abdallah- Prétceille et L. Porcher partagent le même avis, selon eux,

La littérature c'est l'humanité de l'homme, son espace personnel. Elle rend compte à la fois de la réalité, du rêve, du passé et du présent, du matériel et du vécu. Il faudrait probablement qu'elle s'enseigne sous des formes neuves, inédites, correspondant aux besoins des hommes d'aujourd'hui. (M. Abdallah-Prétceille et L. Porcher, 1996 : 138)

Dans ces conditions, il est question non pas de savoir s'il faut didactiser la littérature ou pas, mais l'on s'interroge plutôt sur les finalités et les démarches adoptées à cet enseignement.

Pour H. BESSE le texte littéraire est perçu comme un lieu où se dévoilent divers exploits de l'homme. Il stipule que :

Parce qu'en lui la langue travaille et est travaillée plus que dans tout autre texte, parce que sa facture lui assure une relative autonomie par rapport à ses conditions de production et de réception, parce qu'il est l'un des lieux où s'élaborent et se transmettent les mythes et les rites dans lesquels une société se reconnaît et se distingue des autres, le texte littéraire nous paraît particulièrement approprié à la classe de français langue étrangère. " (H. Besse (1991), cité par F. Migeot (2003 : 20)

En effet, le texte littéraire, unique en son genre, de par les divers attributs dont il est couronné, il demeure le support providentiel capable de satisfaire les nouveaux besoins de l'école dont la noble mission consiste à former un citoyen conscient du vivre ensemble.

D'ailleurs, le programme de français en Algérie encourage l'exploitation de ce genre de supports et incite les concepteurs à le valoriser. La section suivante met en relief le corpus de textes littéraires étudiés dans les manuels de français du cycle moyen en Algérie.

\subsection{Présence des textes littéraires dans les manuels}

\subsubsection{Les supports textuels}

30 Le décompte effectué nous a permis de dégager le taux des textes littéraires présents dans les manuels du moyen et leur proportion par rapport au vaste champ des supports textuels à savoir : le texte scientifique, guide touristique, les articles de presse, le texte historique, etc. 
Tableau 2 : Répartition des supports textuels présentés dans les manuels du moyen

\begin{tabular}{|l|l|l|}
\hline Textes supports & Occurrences & Pourcentage \\
\hline Textes littéraires & 132 & $54,54 \%$ \\
\hline Textes scientifiques & 37 & $15,28 \%$ \\
\hline Articles de presse & 41 & $16,94 \%$ \\
\hline Textes historiques & 16 & $6,61 \%$ \\
\hline Guides touristiques & 16 & $6,61 \%$ \\
\hline
\end{tabular}

Le recensement effectué révèle que les textes littéraires sont représentés par 132 occurrences, soit $54,54 \%$ de l'ensemble des supports textuels dénombrés dans les manuels inventoriés. Les textes scientifiques (dits expositifs) sont au nombre de 37, soit $15,28 \%$ de la totalité des textes contre 41 occurrences pour les articles de presse, soit $16,94 \%$. Les textes historiques et les guides touristiques arrivent en quatrième lieu. Ils sont représentés chacun par 16 textes, soit 6,61 \% de l'ensemble des extraits recensés.

Les résultats obtenus nous permettent de constater une remarquable réhabilitation du support littéraire dans les manuels de FLE au moyen.

\subsubsection{La nationalité littéraire}

Dans le but de mieux approfondir notre recherche, nous avons repéré et classé les textes littéraires par auteur :

Tableau 3 : Répartition des textes littéraires recensés par auteur

\begin{tabular}{|l|l|l|l|}
\hline Nom de l'auteur & Occurrences & Nom de l'auteur & Occurrences \\
\hline Antoine Saint Exupéry & 4 & Kristoff Valla & 1 \\
\hline Les mille et une nuits & 3 & Pierre Benoît & 1 \\
\hline Les frères Grimm & 4 & H. G. Wells & 1 \\
\hline A. Pouchkine & 1 & Légende Algérienne & 1 \\
\hline Henri Gaugaud & 1 & André Demaison & 1 \\
\hline Taous Amrouche & 2 & Robert Lawrence Stine & 1 \\
\hline Carlo Collodi & 1 & Abderrahmane Zakad & 1 \\
\hline Andersen & 1 & Simone De Beauvoir & 1 \\
\hline Eric Voisin & 1 & Alphonse de Lamartine & 1 \\
\hline
\end{tabular}




\begin{tabular}{|c|c|c|c|}
\hline Jean Muzi & 1 & Michel Leiris & 2 \\
\hline Conte Japon & 1 & Alexandre Dumas & 1 \\
\hline Conte Egyptien & 1 & Marcel Pagnol & 2 \\
\hline Conte africain & 1 & Marcel Proust & 1 \\
\hline Conte hawaïen & 1 & Léon Tolstoï & 1 \\
\hline Conte algérien & 2 & Jaques Charpentreau & 1 \\
\hline Conte Russe & 1 & Gabriel voisin & 1 \\
\hline Conte Chinois & 3 & Fernand Gregh & 1 \\
\hline Conte Malien & 1 & Edmond De Amicis & 1 \\
\hline Jean De Lafontaine & 15 & Camara Laye & 1 \\
\hline Ésope & 5 & Ahmed Azeggagh & 1 \\
\hline Ibn El Mokafâa & 3 & Mouloud Féraoun & 4 \\
\hline Jean Muzi et Gérard Franquin & 1 & Rabah Belamri & 1 \\
\hline Colette & 1 & Azouz Begag & 1 \\
\hline Maxence Van Der Meersch & 1 & Fadhma Ait Mansour & 1 \\
\hline Roselyne Morel & 2 & Jules Vallès & 1 \\
\hline Ray Bradbury & 3 & Victor Hugo & 4 \\
\hline Dino Buzatti & 1 & Alphonse Daudet & 1 \\
\hline D. Mornet & 1 & Albert Camus & 2 \\
\hline Légende Allemande & 1 & Louis Tournier & 1 \\
\hline Guy De Maupassant & 1 & Mohammed Dib & 2 \\
\hline J. M. Le Clezio & 1 & J.P. Chabrol & 1 \\
\hline Jules Renard & 1 & William Chapman & 1 \\
\hline Jean Giono & 4 & Anna Greki & 1 \\
\hline Paul Fort & 1 & Assia Djebar & 1 \\
\hline Jean Louis Curtis & 1 & Tahar Ben Jelloun & 1 \\
\hline Georges Duhamel & 1 & Nazim Hikmet & 1 \\
\hline
\end{tabular}




\begin{tabular}{|l|l|l|l|}
\hline José Mauro de Vasconcellos & 1 & Paul Verlaine & 1 \\
\hline Alain Serres & 1 & Rabia Ziani & 2 \\
\hline Roahld Dahl & 2 & Pierre Gamarra & 1 \\
\hline Henri Bosco & 1 & Arnold Lobel & 1 \\
\hline Eugène Labiche & 1 & & \\
\hline
\end{tabular}

Un examen des auteurs présents dans les quatre manuels nous révèle une présente très manifeste des auteurs étrangers (particulièrement des Français) mais très peu d'auteurs algériens d'expression française. Ainsi, on constate que les écrivains maghrébins sont très peu sollicités en classe de français du collège.

\subsubsection{Les rubriques choisies}

Dans chaque manuel, il y a trois projets. Chaque projet est subdivisé en deux ou trois séquences ${ }^{1}$. Celles-ci se répartissent en rubriques ou étapes.

De l'ensemble des textes disponibles dans les manuels pour l'enseignement/ apprentissage de la lecture, nous avons compté le pourcentage de présence des textes littéraires dans les différentes rubriques (étapes) des manuels.

Le tableau ci-après donne une idée précise sur le pourcentage de présence du texte littéraire sur le nombre des textes littéraires par manuel et par rubrique.

Tableau 4 : Présence du texte littéraire, en pourcentages, dans les différentes rubriques des manuels

\begin{tabular}{|l|l|l|l|l|}
\hline Niveau d'enseignement & 1 AM & 2 AM & 3 AM & 4 AM \\
\hline Textes littéraires en général & $45,45 \%$ & $100 \%$ & $55,17 \%$ & $73,68 \%$ \\
\hline Textes littéraires de la rubrique compréhension de l'écrit & $0 \%$ & $100 \%$ & $44,44 \%$ & $55,55 \%$ \\
\hline Textes littéraires de la rubrique lecture- plaisir & $100 \%$ & $100 \%$ & $76,92 \%$ & $100 \%$ \\
\hline Textes littéraires de la rubrique évaluation- bilan & $33,33 \%$ & $/$ & $28,57 \%$ & $66,66 \%$ \\
\hline
\end{tabular}

36 La lecture de ce tableau révèle que le manuel de la $2^{2 \mathrm{eme}} \mathrm{AM}$ est le plus riche en textes littéraires par rapport aux autres manuels. Le genre de texte narratif représente $100 \%$ des supports proposés à la lecture. Juste après vient le manuel de la $4^{\text {ème }} \mathrm{AM}$ avec $73,68 \%$.

37 Le texte littéraire est présent dans le livre de la 3AM à 55,17\% et dans celui de la $1^{\text {ère }}$ AM à $45 \%$.

Par rapport aux différentes rubriques de lecture, en général, le texte littéraire occupe une place très importante dans la rubrique "lecture-plaisir » avec 92,10\%. Dans l'activité " compréhension de l'écrit ", on le trouve à $52,78 \%$. Tandis que dans la rubrique 
« Évaluation-bilan » le texte littéraire n'est représenté qu’à 37,5 \% des textes proposés. Compte tenu du fait que tous les supports proposés pour la " lecture- plaisir » soient des textes littéraires, le but visé consiste à réconcilier les élèves avec l'activité de la lecture littéraire.

\section{Examen des données recueillies}

Dans notre étude, nous avons opté pour le traitement des textes littéraires disponibles dans les manuels pour l'enseignement/apprentissage de la lecture.

Cette analyse descriptive ayant pour but de déterminer la présence du texte littéraire parmi les supports textuels proposés dans les quatre manuels. Le corpus d'analyse se compose de tous les textes dédiés à la lecture dont l'objectif est de construire un sens à partir d'un document écrit.

Cette construction de sens peut être guidée par un questionnaire de compréhension accompagnant le texte en question pour inciter l'apprenant à relever quelques informations ou d'opérer des inférences preuves de sa compréhension. Dans ce cas, nous avons deux rubriques (étapes) : une se présentant au début de chaque séquence intitulée " compréhension de l'écrit ", l'autre située à la fin de la séquence ou du projet, portant comme titre " révision bonus " dans le manuel de la $1^{\text {ère }} \mathrm{AM}$ et « Évaluation-bilan » dans ceux de la $3^{\text {ème }} \mathrm{AM}$ et $4^{\text {ème }} \mathrm{AM}$.

41 Toujours dans le cadre de la construction du sens à partir de l'écrit, nous pouvons citer des textes d'une longueur plus importante que celle des textes des deux rubriques précédentes mais, par contre, ils ne sont pas suivis de questions. Ces textes sont présentés à l'apprenant en vue d'une lecture de distraction d'où l'intitulé de la rubrique : "Plaisir de lire », dans le manuel de la 1AM, « lecture-plaisir » dans celui de la $2^{\text {ème }} \mathrm{AM}$ et « Lecture récréative » dans les deux manuels de la $3^{\text {ème }} \mathrm{AM}$ et la $4^{\text {ème }}$ AM.

2 Nous tenons à signaler que nous avons écarté les textes exploités pour l'apprentissage exclusif du vocabulaire et de la grammaire ainsi que ceux utilisés comme supports dans le but de préparer l'élève à la production de l'écrit et bien sûr les textes des activités de l'oral.

3 Un examen approfondi des données recueillies nous amène à constater les points suivants :

Un retour effectif de la littérature dans les manuels de français du cycle moyen.

Les manuels utilisent une structuration fixe qui reproduit dans chaque projet le même séquençage. Nous avons nommé ces étapes « rubrique ».

"Une séquence se structure de la façon suivante:

1. La compréhension de l'oral: Des supports sont proposés mais les enseignants peuvent en choisir d'autres plus adaptés au niveau de leur public.

2. La production de l'oral : Elle se fait à partir d'un support (dessin, photo, tableau, vignette de $\mathrm{BD}, . .$.$) et d'un questionnaire.$

3. La compréhension de l'écrit : Les activités proposées permettront à l'apprenant d'apprendre à construire progressivement le sens d'un texte en passant par quatre étapes :

4. - J'observe et j'anticipe.

5. - Je lis pour comprendre (compréhension globale) 
6. - Je relis pour mieux comprendre (compréhension détaillée)

7. - Je retiens l'essentiel (synthèse)

8. Des outils pour dire, lire et écrire : L'étude de chaque point de langue s'appuie sur la lecture d'un corpus (généralement, un texte écrit).

9. Atelier d'écriture (...)

10. Évaluation-bilan (...)

11. Station-projet (...)

12. Lecture récréative (...) » (Guide du manuel de Français, 4ème année moyenne : 13) ce qui le place dans une position d'exemple et de support pédagogique. Le thème est un point grammatical, un champ lexical ou la prononciation d'un phonème. Ce n'est plus le texte littéraire qu'il faut lire mais le point technique qu'il faut déceler dans le texte.

Les documents littéraires peuvent en outre être inclus dans la troisième rubrique dite " Lecture plaisir » où le texte littéraire occupe une place très importante.

51 Revenons à présent à la mise en page des quatre manuels qui rappelons-le, revêt une grande importance sur plusieurs plans. Elle a d'abord une fonction utilitaire pour le lecteur qu'est l'apprenant. Elle a ensuite une fonction de séduction en direction des enseignants, des équipes pédagogiques et également des apprenants. Elle a enfin une fonction de distinction qui permet d'identifier les documents.

Les documents littéraires ne sont pas clairement présentés comme des textes littéraires. Ils sont banalisés. Ils apparaissent parmi d'autres documents sans indication particulière ou précision à propos de leur nature. Ils s'insèrent dans la leçon sans ostentation tout en comportant les mentions nécessaires à l'identification de leur origine et de leur statut. Autrement dit, la littérarité des textes littéraires insérés n'est 
pas affichée au moyen de photographie de l'objet-live où figure l'extrait qui sera exploité.

Ce genre de présentation affiche clairement le statut originel du texte et indique dès le premier regard qu'il est extrait d'une œuvre littéraire. Nous pouvons donc dire qu'il s'agit, dans ce cas, d'un manque de mise en situation car le texte est censé rappeler une situation authentique de lecture.

54 Les concepteurs de ces manuels optent pour une présentation totalement différente qui banalise le document littéraire. En effet, le texte est toujours accompagné par les mentions minimales nécessaires, le nom de l'auteur ainsi que le titre de l'œuvre dont il est extrait, mais aucune autre indication n'est donnée. À ce propos Cicurel (1991: 129) souligne que » les textes à lire sont imposés, ils sont souvent tronçonnés, le lecteur est privé de l'incipit, de la suite du récit" ".

On a relevé aussi un certain discrédit du fait littéraire. En effet, le texte littéraire est très souvent inséré dans les pages du manuel tout comme un exercice de grammaire. D'ailleurs, il est convoqué pour réaliser des tâches uniquement linguistiques où sa littérarité n'est point exploitée.

En outre, la consigne de l'exercice ne précise pas l'origine du texte littéraire en question, elle n'est pas exploitée, et il n'est pas demandé aux apprenants de comprendre le sujet de l'extrait.

Dans telle situation, l'apprenant doit pouvoir identifier le document qu'il va aborder afin d'utiliser des stratégies de réception adéquates. Il peut le faire ici puisque le nom de l'auteur et le titre du roman sont cités dans la consigne de l'exercice. Il n'y est cependant pas motivé. La mise en page du document littéraire fait oublier la littérarité du texte et une partie des informations est atrophiée. L'écrivain n'est pas contextualisé, il n'y a aucune indication à propos du roman.

57 Ainsi, lorsqu'on insère le texte littéraire dans une rubrique portant sur les documents écrits ou sur la compétence de compréhension écrite, le texte doit être visible et identifiable en tant que tel et en tant que document authentique. Les activités associées accompagnent la réception et permettent de développer des compétences de compréhension écrite qui sont nommées stratégies de lecture ou approche globale.

Concernant le nombre de textes littéraires que nous avons relevé il est plutôt important. Le choix des textes est diversifié, tant du point de vue des auteurs représentés que des genres littéraires choisis. Les écrivains français du XXe et du XXI siècle dominent, comme le genre romanesque.

En revanche, le genre poétique, à titre d'exemple, se trouve souvent marginalisé sans questions d'accompagnement et notes explicatives. En même temps, la grande majorité des poèmes se trouve confinée à la fin du manuel (réservés pour la lecture récréative).

Nous avons constaté que le genre théâtral est quasi inexistant (une seule présence relevée dans le manuel de $4^{\text {ème }} \mathrm{AM}$ ).

59 En somme, les tableaux statistiques le montrent bien, la littérature est présente dans la majorité des manuels. Cette observation confirme qu'il ne semble plus possible aujourd'hui de négliger ce document dans l'intégralité d'un ensemble pédagogique. Les choix sont divers, tant pour la forme du texte que pour sa thématique, et les différents genres littéraires sont représentés. Les concepteurs des manuels concentrent leurs choix vers les auteurs contemporains afin de proposer une image récente de la littérature, ancrée dans le présent et plus proche des apprenants. 
60 Dans les manuels que nous avons observés, les portes se sont ouvertes et les œuvres maghrébines. Cette place accordée à la francophonie correspond en partie à la position actuelle du champ institutionnel algérien qui veut réhabiliter plusieurs écrivains nationaux.

La littérature apparaît à présent être un support pédagogique adéquat et polyvalent dans le cadre de l'enseignement et de l'apprentissage du français langue étrangère.

En paraphrasant E. Riquois (2009) qui estime que le texte littéraire en tant qu'objet appartenant au patrimoine culturel d'un pays, il permet d'aborder des domaines de connaissances culturelles qui peuvent constituer des modes d'identification sociale.

Les manuels de français du moyen sont des outils intéressants pour observer les usages de la littérature dans les pratiques pédagogiques actuelles. Variés, très utilisés, ces ouvrages donnent des indications qui ne se confondent pas avec les pratiques effectives des enseignants mais permettent de déterminer les caractéristiques des textes littéraires potentiellement utilisés en classe. Les manuels proposent aussi des exploitations pédagogiques qui marquent des représentations de la littérature d'un autre âge.

La répartition de l'appartenance générique des textes exploités manifeste une nette orientation vers les textes narratifs : romans et contes, fables, légendes et romans de science-fiction.

Type générique connu des apprenants, la narration semble être le genre le plus adapté à une exploitation pédagogique à tous les niveaux.

61 En définitive, la littérature semble constituer un champ d'exploitation pédagogique potentiellement riche et susceptible de donner lieu à des activités originales et intéressantes pour les apprenants. Qu'il s'agisse du domaine de l'interculturel ou de la compétence de lecture, le texte littéraire permet de favoriser chez l'apprenant le développement d'une autonomie qui lui sera indispensable en dehors de la salle de classe.

\section{BIBLIOGRAPHIE}

ABDALLAH-PRETCEILLE, M. et PORCHER, L., Éducation et communication interculturelle. Paris : PUF, 1996.

BESSE. H., « Quelques réflexions sur le texte littéraire et ses pratiques dans l'enseignement du français langue seconde ou langue étrangère " Trèfle, $\mathrm{n}^{\circ}$ 9, Lyon, 1989.

CICUREL, F. Lectures interactives en langues étrangères. Paris : Hachette FLE, 1991.

COLLÈS, L., Littérature comparée et reconnaissance interculturelle. Bruxelles : De Boeck, 1994.

COLLÈS, L., "Une Pratique littéraire de l'interculturalité”. In : DUFAYS, J. L., GEMENNE, L. et LEDUR, D. (dir.). Pour une lecture littéraire 2 : bilan et confrontations. Actes du colloque "La lecture littéraire en classe de français : quelle didactique pour quels apprentissages ?", LouvainlaNeuve, 3-5 mai 1995. Bruxelles : De Boeck Duculot. p. 227-233, 2005. 
DAKHIA, A. (2005). Dimension pragmatique et ressources didactiques d'une connivence culturelle en FLE. Thèse de doctorat. Université de Batna.

Guide du manuel de Français de 4ème année moyenne

GERFAUD, J.-P et TOURREL, J.-P. (2004). Pour une lecture anthropologique de l'œuvre littéraire, Thèse de Doctorat, Université Lumière Lyon 2, 2000.

LOUIS, V., "Interactions verbales et communication interculturelle en FLE : de la civilisation française à la compétence (inter) culturelle, Éditions EME, 2009.

PORCHER, L., Manières de classe. Paris : Didier, 1987.

RIQUOIS E., (2009). Pour une didactique des littératures en français langue étrangère: Du roman légitimé au roman policier, Thèse de Doctorat Université de Rouen.

SÉOUD, A., Pour une didactique de la littérature. Paris : Hatier-Didier : CREDIF, 1997.

\section{NOTES}

1. La séquence est l'ensemble des séances qui participent au même objectif d'apprentissage. (Document d'accompagnement du manuel de la 4AM : 38)

\section{RÉSUMÉS}

Cet article s'inscrit dans la réflexion sur l'utilisation des textes littéraires dans les manuels du cycle moyen. Ce travail a pour but de réfléchir sur quelques moyens possibles pour mieux approcher les textes littéraires en classe de FLE de nos jours.

Les observations menées sont centrées sur, d'une part, les textes littéraires répertoriés et l'observation des activités proposées pour l'exploitation pédagogique, d'autre part.

Les résultats montrent que la littérature est bien présente dans les manuels du moyen, où elle est traitée en tant que support pour l'assimilation des contenus linguistiques. Toutefois, le souci se situe au niveau des objectifs d'apprentissage alloués à cet emploi pédagogique de la littérature.

Notre proposition tient des stratégies de l'approche interculturelle et anthropologique qui envisage le texte littéraire comme une passerelle dans la relation avec autrui. Elle s'articule autour d'une didactique de la littérature qui n'a pas pour objectif de réduire le texte littéraire à une représentation socio-historique et faire de lui un témoin d'une évolution historique des mentalités, mais bien plutôt d'affirmer l'évolution humaine comme une totalité et un tout.

This article is part of the reflection on the use of literary texts in textbooks of the middle cycle. This work aims to reflect on some possible ways to better approach the literary texts in FLE classroom today.

The observations made focus on, on the one hand, the listed literary texts and the observation of the proposed activities for the educational exploitation of literary texts, on the other hand.

The results show that the literature is present in the manuals of the medium, where it is treated as a support for the assimilation of linguistic contents. That said, there is concern about the learning objectives allocated to this pedagogical use of the literature. 
Our proposal holds strategies of the intercultural and anthropological approach that considers the literary text as a bridge in the relationship with others. It is based on a didactic of literature which does not aim to reduce the literary text to a socio-historical representation and to make of it a witness of a historical evolution of the mentalities, but rather to affirm human evolution as a totality and a whole.

\section{INDEX}

Keywords : language teaching/learning, didactics of literature, interculturality, otherness, universal-singular

Mots-clés : enseignement/apprentissage des langues, didactique de la littérature, interculturalité, altérité, universels- singuliers

\section{AUTEUR}

\section{FATAH ABDELOUHAB}

Université Abderrahmane Mira, Bejaia, Algérie 